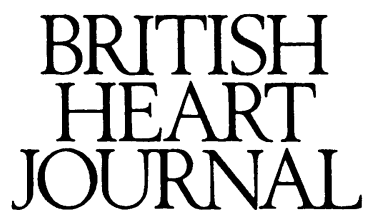

\title{
Should we get up in the morning? Observations on circadian variations in cardiac events
}

The recent fascination with circadian patterns in cardiovascular disease processes has led to a plethora of papers on the subject. But why do such variations occur and what does it all mean? In this article we analyse the findings of recent studies and discuss the clinical implications of circadian variations in cardiovascular disease processes.

\section{Myocardial infarction, sudden cardiac death, and} stroke

Several studies show a significant peak in the incidence of acute myocardial infarction in the morning waking hours. ${ }^{1-4} \mathrm{Hjalmarson}$ et al, in a study of over 4500 patients with acute myocardial infarction, reported a primary morning peak in events and a lesser evening peak (fig 1), ${ }^{1}$ confirming the findings of Muller and colleagues. ${ }^{2}$ In the ISAM (intravenous streptokinase in acute myocardial infarction) trial and the Physicians Health Study there was a significant morning peak in acute myocardial infarction. ${ }^{34}$ Also Thompson and his colleagues found a primary peak in the morning and a lesser one at midnight for onset of chest pain. ${ }^{5}$ Reports assessing the time of sudden cardiac death confirm the same morning peak in incidence. ${ }^{67}$ This is not surprising because many sudden deaths result from acute myocardial infarction. The incidence of ischaemic stroke has also been shown to be highest in the morning. ${ }^{8}$

\section{Why "thrombosis and death at dawn"?}

To determine why these critical cardiac events cluster in the morning waking hours, we must look at the variables that predispose to such events. Myocardial ischaemia can now be assessed easily during daily routines by ambulatory ST segment monitoring, and in patients with coronary disease it has been amply demonstrated that there is a considerable increase in ischaemic activity (both silent and

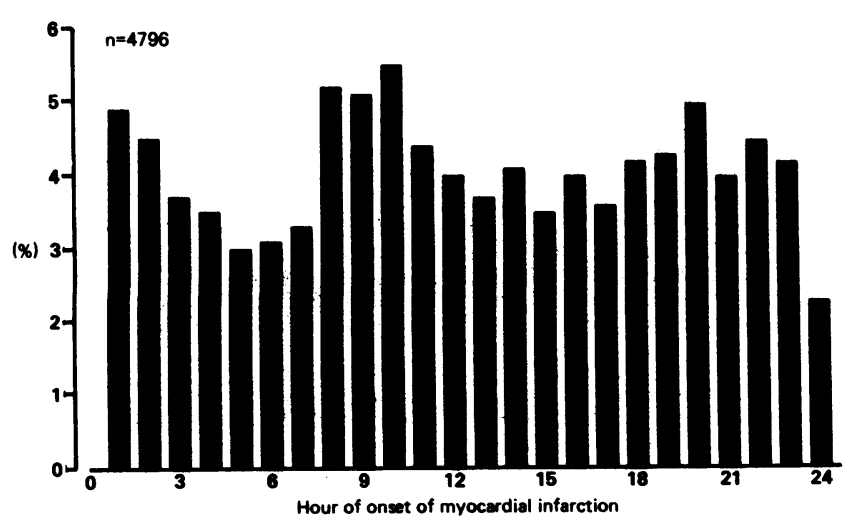

Figure 1 Distribution of hours of symptom onset of myocardial infarction ( $n=4796$ ) by six-hour intervals (reproduced with permission of Hjalmarson A, et al. Circulation 1989;80:267-75). symptomatic) during the morning waking hours, ${ }^{910}$ which may predispose to the end points of coronary disease. The variables that we seek to control by medical treatment in patients with coronary artery disease include heart rate, blood pressure, coronary blood flow, and the thrombotic state. It has now been shown that a surge in heart rate $e^{1112}$ and blood pressure ${ }^{1213}$ at this time coincides with a peak in catecholamine release. ${ }^{14}$ These combined alterations increase myocardial oxygen demand, coronary and systemic vasoconstriction, and the risk of ventricular arrhythmia. The surge in blood pressure increases shear stresses at the sites of coronary stenoses which may lead to plaque fissuring with its resultant complications. Coronary blood flow is also significantly less in the morning than in the afternoon, ${ }^{15}$ resulting in a reduction in myocardial oxygen supply. There is a peak in platelet stickiness in the morning ${ }^{1416}$ and a trough in the fibrinolytic state. ${ }^{17} 18$ Thus the tendency to thrombosis is greatest at this time.

\section{Should we stay in bed until after lunch?}

Staying in bed in the morning is not likely to be cardioprotective; a recent study by Goldberg et al $^{19}$ tends to confirm this. They showed a peak incidence $(23 \%)$ of onset of symptoms of acute myocardial infarction in the first hour after awakening rather than, less specifically, in the morning hours (fig 2). ${ }^{19}$ Ridker et al's study also showed this association with awakening. ${ }^{4}$ Rocco and colleagues showed that transient ischaemia is more common in the two hours after awakening. ${ }^{9}$ The peak in platelet stickiness seems to relate not particularly to time but to getting up. ${ }^{16}$ Though blood pressure begins to rise before waking the major surge results from the start of daily activities. ${ }^{13}$ Finally, members

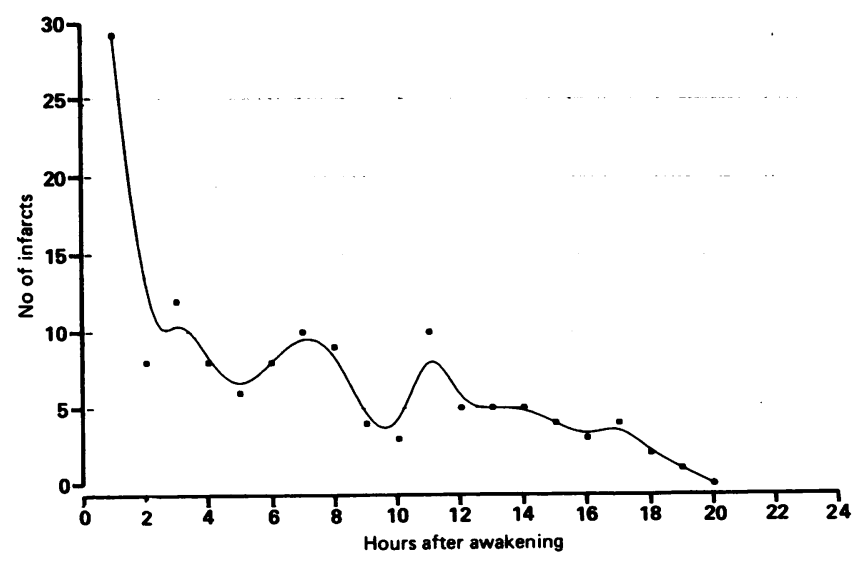

Figure 2 Time of onset of initial symptoms of acute myocardial infarction after awakening (reproduced with permission of Goldberg $R J$, et al. Am J Cardiol 1990;66:140-4). 


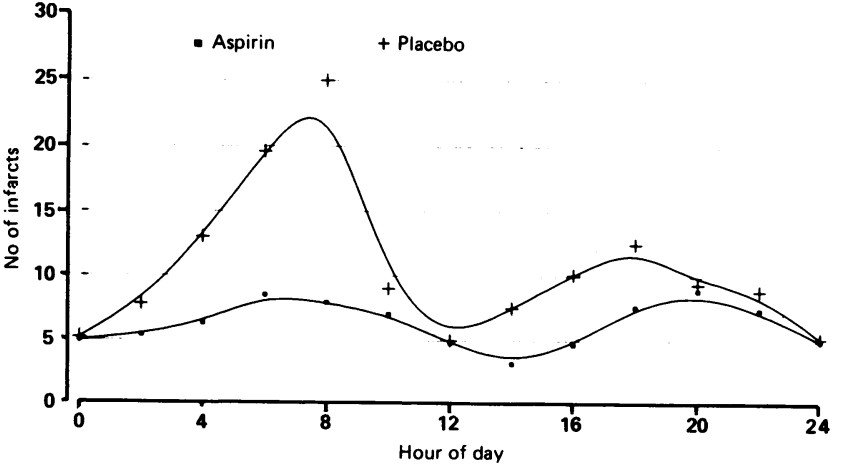

Figure 3 Estimated time and frequency of myocardial infarction onset among 11034 subjects at risk in the aspirin group and 11037 subjects at risk in the placebo group (reproduced with permission of Ridker PM, et al. Circulation 1990;82:897-902).

of a symphony orchestra who had an unusual temporal pattern of work showed significant alterations in the circadian pattern of heart rate, ${ }^{20}$ which presumably reflects similar changes in catecholamine release and blood pressure. Because most people get up in the morning it is difficult to study circadian patterns in cardiac disease. If we all got up at noon the peak in cardiovascular events would probably simply be shifted to the afternoon. Thus the concept of "thrombosis and death at dawn" could be more aptly described as "thrombosis and death on arising". The potential cardiovascular benefits of not getting up at all would surely be far outweighed by a reduced quality of life and the complications of prolonged bed rest.

\section{Can we modify these circadian patterns?}

In theory, any pharmacological intervention that alters heart rate, blood pressure, coronary blood flow, or the thrombotic state favourably for the coronary patient should have its greatest beneficial effect in the morning waking hours. This has been confirmed in various studies. Muller $e t$ al noted that there was no morning peak in acute myocardial infarction in those receiving $\beta$ blocking agents, ${ }^{2}$ and this was confirmed in the ISAM trial ${ }^{3}$ and in Hjalmarson's report. ${ }^{1}$ Furthermore, $\beta$ blockers eradicate the morning peak in ischaemia, ${ }^{1021}$ and in the Beta-Blocker Heart Attack Trial the incidence of sudden death was significantly reduced in the group receiving propranolol, with this

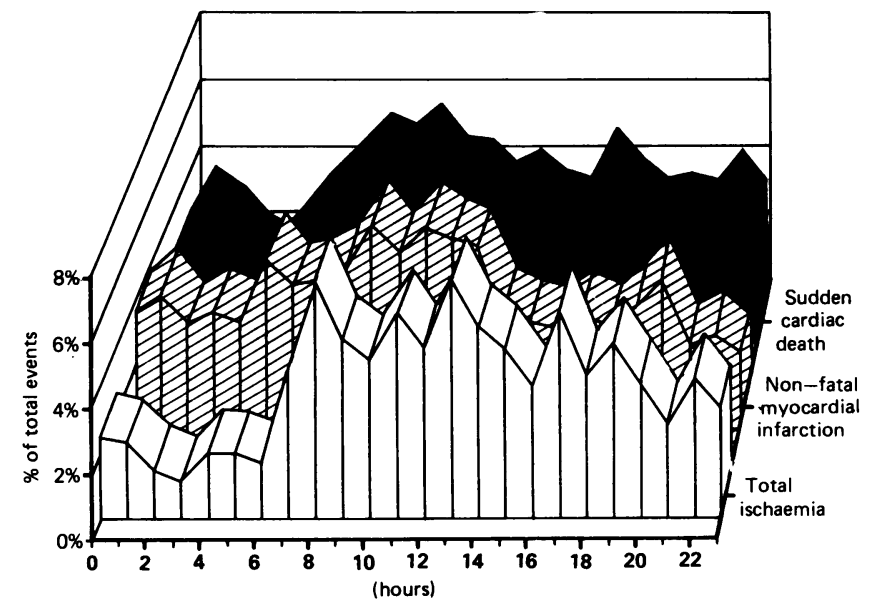

Figure 4 Ciradian variation in transient myocardial ischaemia, nonfatal myocardial infarction, and sudden cardiac death (reproduced with permission of Mulcahy D, et al. Lancet 1988;ii:755-9). beneficial effect being almost totally confined to the morning hours. ${ }^{22}$ Calcium antagonists do not seem reliably to alter the circadian pattern of ischaemia ${ }^{10}$ or acute myocardial infarction, ${ }^{3}$ suggesting that alteration in coronary vasomotor tone is not the predominant precipitating factor in these morning events: indeed it has been clearly shown that those patients with extreme alteration in coronary vasomotor tone (coronary spasm) have a peak in ischaemic episodes at night. ${ }^{23} \mathrm{~A}$ recent study of high doses of nifedipine, however, suggests that the morning surges in ischaemia can be prevented. ${ }^{24}$

Although Willich et al found no alteration in the "classical" circadian pattern of infarction in those receiving aspirin, ${ }^{3}$ the recently published Physicians Health Study of over 22000 subjects showed a significant reduction in the incidence of non-fatal myocardial infarction in those receiving aspirin, and showed that this beneficial effect resulted largely from a reduction in events in the morning waking hours, the reported time of peak platelet stickiness (fig 3). ${ }^{4}$

\section{Clinical implications}

Only one general fact has emerged from the study of circadian patterns in cardiovascular function: the time soon after awakening is a particularly dangerous one for the coronary patient (fig 4). This is not surprising because we know that all the variables that we seek to control and modify with medical or surgical intervention act against the patient with coronary disease primarily at this time. We now know that we can modify the circadian patterns of ischaemia and the end points of cardiac disease particularly with the use of $\beta$ blockade, though whether this translates into an improved outlook for the patient is not clear. Aspirin modifies the morning peak in infarction and does improve patient outlook, suggesting a strong relation between platelet activity and acute myocardial infarction. The morning peak in "cardiovascular disease activity" and the alteration in circadian patterns in those with different temporal patterns of work emphasise the potential importance of 24 hour protection for the cardiac patient. Further study is required in patients with coronary artery disease and altered temporal patterns of work to establish whether the end points of coronary disease are related primarily to the sleep-wake period or to identifiable periods of increased activity or stress during daily life.

DAVID MULCAHY HENRY PURCELI

Royal Brompton National Heart and Lung Hospital,

Sydney Street

London SW3 $6 N P$

1 Hjalmarson A, Gilpin EA, Nicod P, et al. Differing circadian patterns of symptom onset in subgroups of patients with acute myocardial infarction. symptom onset in subgroups

2 Muller JE, Stone PH, Turi ZG, et al. Circadian variation in the frequency of onset of acute myocardial infarction. $N$ Engl J Med 1985;313:1315-22.

3 Willich SN, Linderer T, Wegscheider K, Leizorowicz A, Alamercery I, Schroder R. Increased morning incidence of myocardial infarction in the ISAM study: absence with prior beta-adrenergic blockade. Circulation 1989;80:853-8.

4 Ridker PM, Manson JE, Buring JE, Muller JE, Hennekens CH. Circadian variation of acute myocardial infarction and the effect of low-dose aspirin in a randomized trial of physicians. Circulation 1990;82:897-902.

5 Thompson DR, Sutton TW, Jowett NI, Pohl JEF. Circadian variation in the frequency of onset of chest pain in acute myocardial infarction. Br Heart $J$ 1991;65:177-8.

6 Muller JE, Ludmer PL, Willich SN, et al. Circadian variation in the frequency of sudden cardiac death. Circulation 1987;75:131-8.

7 Willich SN, Levy D, Rocco MB, Tofler GH, Stone PH, Muller JE. Circadian variation in the incidence of sudden cardiac death in the Framingham Heart Study population. Am J Cardiol 1987;60:801-6.

8 Marler JR, Price TR, Clark GL, et al. Morning increase in onset of ischaemic stroke. Stroke 1989;20:473-6.

9 Rocco MB, Barry J, Campbell S, et al. Circadian variation of transient myocardial ischemia in patients with coronary artery disease. Circulation 1987;75:395-400 
10 Mulcahy D, Keegan J, Cunningham D, et al. Circadian variation of total ischaemic burden and its alteration with anti-anginal agents. Lancet 1988;ii:755-9.

11 Northcote RJ, Canning GP, Ballantyne D. Electrocardiographic findings in male veteran endurance athletes. Br Heart $J 1989 ; 61: 155-60$.

12 Millar-Craig MW, Bishop CN, Raftery EB. Circadian variation of blood pressure. Lancet 1978;i:797-9.

13 Furlan R, Guzzetti S, Crivellaro W, et al. Continuous 24 hour assessment of the neural regulation of systemic arterial pressure and $R R$ variabilities in ambulant subjects. Circulation 1990;81:537-47.

14 Tofler GH, Brezinski D, Schafer AI, et al. Concurrent morning increase in platelet aggregability and the risk of myocardial infarction and sudden death. N Engl J Med 1987;316:1514-8. 15 Fujita M, Franklin D. Diurnal changes in coronary blood flow in conscious
dogs. Circulation 1987;76:488-91.

16 Brezinski DA, Tofler GH, Muller JE, et al. Morning increase in platelet aggregability. Association with assumption of the upright posture. Circulation 1988;78:35-40.

17 Andreotti F, Davies G, Hackett DR, et al. Major circadian fluctuations in fibrinolytic factors and possible relevance to time of onset of myocardial fibrinolytic factors and possible relevance to time of onset of myocardial
infarction, sudden cardiac death and stroke. Am J Cardiol 1988;62:635-7.
18 Angleton $P$, Chandler WL, Schmer G. Diurnal variation of tissue-type plasminogen activator and its rapid inhibitor (PAI-1). Circulation 1989;79:101-6.

19 Goldberg RJ, Brady P, Muller JE, et al. Time of onset of symptoms of acute myocardial infarction. Am J Cardiol 1990;66:140-4.

20 Mulcahy D, Keegan J, Fingret A, et al. Circadian variation of heart rate is affected by environment: study of continuous electrocardiographic mon-

21 Cohn PF, Lawson WE. Effects of long-acting propranolol on AM and PM peaks in silent myocardial ischemia Am J Cardiol 1989;63:872-3.

22 Peters RW, Muller JE, Goldstein S, Byington R, Friedman LM. Propranolol and the morning increase in the frequency of sudden cardiac death (BHAT Study). Am J Cardiol 1989;63:1518-20.

23 Nademanee K, Intarachot V, Josephson MA, Singh BN. Circadian variation in occurrence of transient overt and silent myocardial ischemia in chronic stable angina and comparison with Prinzmetal angina in men. Am Cardiol 1987;60:494-8.

24 Nesto RW, Phillips RT, Kett KG, McAuliffe LS, Roberts M, Hegarty P. Effect of nifedipine on total ischemic activity and circadian distribution of myocardial ischemic episodes in angina pectoris. Am J Cardio 1991;67:128-32. 